\title{
The correlation of duration of cervical flexion per day with neck disability index scores in office workers: a cross sectional study
}

\section{Zhang Jinlong}

Hefei BOE Hospital

Fang Yunyun ( $\nabla$ cdfk22@tom.com )

Jiangning Hospital of Nanjing Medical University

Gao Lijie

Sichuan University West China Hospital

Chen Jian

China University of Science and Technology

Wang Cheng

China University of Science and Technology

He Hongchen

Sichuan University West China Hospital

Luo Qinglu

Guangzhou Medical University

Research article

Keywords: DCF, office workers, cervical spondylosis, NDI, USP

Posted Date: January 19th, 2021

DOl: https://doi.org/10.21203/rs.3.rs-34223/v4

License: (c) (i) This work is licensed under a Creative Commons Attribution 4.0 International License. Read Full License 


\section{Abstract}

Background: An increasing number of office workers complain of neck pain after extended smart phone use. We conducted this study to examine the correlation between the duration of cervical flexion per day (DCF) during smart phone use and neck disability index scores (NDI) scores of office workers in China.

Methods: In this cross-sectional study, 1791 individuals responded to an on line questionnaire. For our study, we included responses of 498 [27.8\%] office workers. Excluded from the study were 1293 [72.9\%] individuals including building workers, students, non-office workers, and those who did not complete the questionnaire. Participants completed an online questionnaire survey from May 29, 2019 to April 10, 2020. The NDI scores were used to evaluate cervical symptoms. Linear regression, threshold saturation effect analysis, t-test, and Pearson's chi-square tests were used to analyze the data.

Results: We analyzed questionnaire results of the 498 office workers. The t-test showed no significant differences among the gender groups for age, working age, and NDI scores ( $P>0.05)$. While low back pain had a strong correlation with NDI scores [ $\beta(95 \% \mathrm{Cl}): 2.40(1.49,3.31), P<0.0001]$. When adjusting for age, working age, and low back pain covariates, DCF had a positive correlation with NDI scores. In addition, DCF had a curve line correlation with NDI scores-a monotone increasing relationship; the fold point was 6 $(P<0.05)$.

Conclusions: DCF had a positive curve line correlation with the NDI scores suggesting that office workers should limit DCF to a maximum of 6 hours to decrease NDI scores.

\section{Background}

An increasing number of office workers are seeing doctors for complaints of neck pain. In our clinic, we have found that the complaints appear to relate to the amount of time that the head is tilted downward while using smart phones or other electrical devices. Cervical spondylosis (CS) refers to the clinical symptoms caused by cervical instability, often due to degeneration of the cervical discs and intervertebral joints, and cervical hyperplasia, which can stimulate or compress the surrounding nerve roots, vertebral arteries, sympathetic nerves, spinal cord, and other tissues [1-3]. In work and in life, there has been a dramatic increase in the use of computers, smart phones, and other electronic devices. In recent years, smart phones have become more and more powerful. In addition to the call function, smart phones can be used to surf the Internet, can be used as a tool for learning or entertainment, and even for office use. More and more office workers use them for office activities. Furthermore, studies have shown an increase in work and academic pressures. Many individuals work or study for many hours at a time. This can lead to cervical or other symptoms $[4,5]$.

The International Labor Organization has classified shoulder and neck pain as occupational diseases since 1960. On July 30, 2019, the Advanced Committee of Healthy China Action announced that diseases such as CS, periarthritis of the shoulder, low back pain, bone hyperplasia, and sciatica are preventable diseases among laborers. Among these diseases, the incidence of CS is the highest. According to the 
2015 data released by the European Agency for Safety and Health at Work, the incidence of CS from 2000 to 2012 showed an annually increasing trend, with rates in some countries as high as $46.3 \%$ [6]. In China's modern era of rapid economic development, increasingly more professional office workers have career-related diseases such as CS.

Two region-specific questionnaires for the cervical spine are the Neck Disability Index (NDI) and the Neck Pain and Disability Scale (NPDS) [7]. The NDI is designed to measure activity limitations due to neck pain and disability [8-10], whereas the NPDS measures problems with neck movements, neck pain intensity, effect of neck pain on emotion and cognition, and the level of interference during life activities [11]. NDI is easier for the lay public to understand and considered a responsive measure for neck pain and acute whiplash injury [12], we chose this scale for our analysis.

It is obvious that duration of DCF is associated with increased NDI scores. In today's information age, it is almost impossible for office workers to give up cervical flexion activities to reduce NDI scores. However, they do not know what range of DCF needs to be restricted. In order to improve the quality of life among office workers, we sought to investigate the correlation between the time of cervical flexion (DCF) and the NDI among office workers of China, and whether the smart phone use had a negative effect on the cervical spine. As far as we know, few scholars pay attention to the relationship between the DCF of smart phone use and NDI scores.

\section{Methods}

\section{Data collection}

We used the Questionnaire Star (https://www.wjx.cn/) to conduct a real-time online cross-sectional study, and used the WeChat and QQ (https://weixin.qq.com/, https://qq.com/) to distribute and collect questionnaires. The survey was conducted from May 29, 2019 to April 10, 2020. Respondents came from nationwide; the recruitment target was office workers and self-reported variables and covariates. The NDI was used to assess the perceived pain and disability related to the cervical spine. In order to eliminate the possibility of repeated measurement, the duplicate data under the same IP address has been deleted and only one data record were kept for analysis of each IP.

\section{Design and setting}

Cross-sectional

\section{Statistical methods}

As far as we know, no similar research has been seen so far, so we did not found the appropriate literature and could not obtain the corresponding value for calculation. Therefore, in this study, we took a purposive approach with no sample estimation. Data were analyzed using the statistical packages $\mathrm{R}$ (R Foundation; http://www.r-project.org; version 3.4.3) , EmpowerStats (www.empowerstats.com; X\&Y Solutions Inc.). 
We performed descriptive statistics for age, working age, DCF, educational degree, activities, low back pain and neck pain and number of participants. Data between sexes were compared using chi square or Fisher's exact test for categorical data and two-sample t-test or Mann-Whitney $\mathrm{U}$ test for continuous data. We estimated beta $(\beta)$ with $95 \%$ confidence intervals (Cls) using multivariate linear regression for continuous data. Generalized additive model were used to visually assess functional relationships between the continuous covariates (DCF and NDI scores) for spline smoothing. We first used spline smooth curve fitting to examine whether the independent variable was partitioned into intervals. We applied segmented regression (also known as piece-wise regression) to conduct threshold saturation effect analysis that was using a separate line segment to fit each interval. We also conducted log likelihood ratio test comparing one-line linear regression model with two-piece-wise linear model. We selected potential confounders in the final models if they changed the estimates of NDI at least $10 \%[13$, $14]$, as seen in supplementary table. The mean \pm standard deviation (SD) and $95 \%$ confidence interval (CI) were used to indicate the size of the value. All $P$-values were two-sided and values below 0.05 were considered statistically significant.

\section{Results}

\section{Flow chart of this study}

A total of 1791 individuals participated in the online questionnaire survey, with 498 (27.81\%) office workers included, and 1293 individuals excluded (5 were under 18 years). Those participants who were excluded were $563(31.34 \%)$ individuals who did not complete the questionnaire and 730 (40.76\%) individuals were no office workers, including 218 (12.17\%) students, 78 (4.36\%) building workers, and 434 (24.23\%) other non-office workers. The flow chart can be seen in Fig 1.

\section{Sociodemographic and clinical characteristics ofthe sample}

The baseline characteristics of all patients are shown in Table 1. The results of the $t$-test showed no significant differences among the gender groups for age, working age, and NDI scores $(P>0.05)$, and the DCF of males was higher than that of females $(P<0.05)$. Pearson's chi-squared test showed that differences in the number of educational degrees in the male and female groups were significant $(P<0.05)$. The activities ratio of smart phone use, using computers, reading books, and using other electronic devices was $63.71 \% / 78.07 \%, 30.65 \% / 18.72 \%, 1.61 \% / 2.14 \%$, and $4.03 \% / 1.07 \%$ in the male and female groups, respectively. Pearson's chi-square tests showed differences were statistically significant $(P<0.05)$. The ratio of participants with neck pain $(74.19 \%$ male, $66.31 \%$ female) and the incidence of low back pain (73.39\% male, $78.07 \%$ female) were high; the difference between males and females was not significant $(P>0.05)$.

\section{Crude correlation associations of DCF, covariates, and NDI scores of the sample}

As seen in Table 2, single factor correlation analysis showed that age and working age did not have a correlation with NDI scores for participants $(P>0.05)$. DCF had a positive correlation with NDI scores 
$(P<0.05)$. Compared with other activities, smart phone use had no positive correlation with NDI scores $(\beta=0.83,95 \% \mathrm{Cl}=-0.07$ to $1.73, P>0.05)$, while low back pain had a strong correlation with NDI scores $(P<0.05)$.

\section{Linear regression for DCF and NDI scores of the sample}

Low back pain factor was the covariates of NDI scores as seen in supplementary table; we also selected age, gender and working age as covariates, on the basis of their associations of outcome of interest. After adjusting for age, working age, and sex covariates, DCF had a positive correlation with NDI scores $(\beta=0.28,95 \% \mathrm{Cl}=0.13$ to $0.43, P<0.05)$, and after adjusting for low back pain $\mathrm{DCF}$ had a strong positive correlation with NDI scores $(\beta=0.26,95 \% \mathrm{Cl}=0.12$ to $0.40, P<0.05)$. (See Table 3 )

\section{Curve line correlation between the DCF and NDI scores of the sample}

Generalized additive models were used to visually assess the DCF and NDI scores relationships. We adjusted for age, sex, working age, and low back pain factors. The DCF had a curve line correlation with NDI scores-a monotone increasing relationship. (See Fig 2)

\section{Analysis of threshold saturation effect between the DCF and NDI scores of the sample}

As seen in Table 4, we performed threshold saturation effect analysis between DCF and NDI scores in participants. The logarithmic likelihood ratio test showed that there was a fold point $(K=6)$ between DCF and NDI scores, and the differences were statistically significant $(P<0.05)$. When DCF was less than 6 hours $(K<6)$, the estimated change in NDI scores was $0.53,95 \% \mathrm{Cl}$ was 0.26 to 0.81 , and the differences were statistically significant $(P<0.05)$. When DCF was greater than 6 hours $(K>6)$, the estimated change in NDI scores was $-0.03,95 \% \mathrm{Cl}$ was -0.33 to 0.26 , and the differences were not statistically significant $(P>0.05)$. The logarithmic likelihood ratio test showed that this fold point was statistically significant $(P<0.05)$.

\section{Discussion}

With recent rapid socioeconomic development, people are more mindful of their health. Increasingly more individuals who work in the office environment are seeking treatment for pain. In China, outpatients with CS are common in rehabilitation medicine, orthopedics, or traditional Chinese medicine. These patients often complain of neck and back pain, and have occasional complaints of dizziness, limb numbness, walking instability, perineal paresthesia, urinary and fecal incontinence, and sexual dysfunction. Such symptoms can have serious impacts on patients' lives and can result in a sharp decline in the quality of life. In a study conducted at the University Clinical Hospital in Olsztyn, Poland between 2011 and 2015, the most frequent MRI-diagnosed diseases were musculoskeletal diseases (58.0\%), cervical disc disorders (12.5\%), and spondylosis (4.6\%). The authors concluded that the significant number of patients presenting with spinal disorders at young ages (31-40 years) pointed to the necessity of introducing methods in school-aged individuals to prevent disorders of the vertebral column [15]. In the present study, 
we also found that participants had a high incidence of neck pain syndrome $(74.19 \%$ for males and $66.31 \%$ for females).

Previous research reported that most patients with CS were in occupations that required working longterm at a desk or work station, where it is common to remain for extended periods of time with the head tilted downward [16-18]. The cervical spine has seven vertebrae, and the joints formed by each vertebra are more flexible. The anatomical features of the neck provide a large range of movement. Overloading the neck musculature can lead to cervical tissue damage. Holding the head in a lowered or flexed posture maintains the neck muscles in a tensile state, which may cause muscle strain if the position is held over a long period of time. This forward flexion posture may lead to static imbalance and accelerate degeneration of the intervertebral discs, small joints, and ligaments of the neck, eventually resulting in CS.

The NDI covers 10 dimensions of neck-specific disability: pain intensity, personal care, lifting, reading, headache, concentration, work, driving, sleeping, and recreation [19]. This is closely correlated with cervical spine function [18], which can reflect neck pain intensity [20] , and can ultimately be used to evaluate the quality of life [21]. Feng et al. [22] analyzed survey data and found that among ultrasound technicians, lowering of the head for a long period of time led to rates of neck discomfort as high as 93.5\%. Gremark Simenson et al. [23] found that the prevalence of CS among ultrasound technicians was $58 \%$, and positively related to the length of time that the technician's head was held in a downward position while working. These results are consistent with those of our study. We found that DCF was positively correlated with the NDI regardless of unadjusted or adjusted covariates. When DCF increasing by 1 hour, NDI will increased by 0.28 or 0.26 score ( $\beta=0.28$ and 0.26 respectively, see table3). Office workers should be mindful of DCF as much as possible. Our study found that there was a fold point $(\mathrm{K}=6)$ between DCF and NDI scores, and the differences were statistically significant $(P<0.01)$. Many patients with CS have symptoms of neuropathy, however most symptoms are relatively benign and ultimately have a good prognosis [24]. The results of our study suggest that when DCF is greater than 6 hours, participants may adapt to neuropathy symptoms related to neck posture, leading to a decrease in symptoms. Even with this adaptation, cervical syndrome can persist. Therefore, we recommend that office workers limit DCF in order to decrease cervical syndrome.

In the 21 st century, the rapid development of technology has resulted in the widespread use of electronic devices, leading to a near-universal presence of computers and smart phones in many countries, including China. One study found active arthritic changes caused by repetitive strain injury from excessive text messaging using smartphones[25]. Another study reported that smartphone use induced a more flexed posture on the neck and trunk than other visual display terminal work[26] Pain and fatigue worsened with longer smartphone use, and the authors suggested correct posture and breaks of at least 20 minutes when using smartphones[27]. Although our research found that using flexed neck postures to use smart phones and for other activities had no effect on NDI scores, DCF was positively correlated with NDI scores. We suggest that office workers need to control DCF to reduce NDI scores when using smart phones or engaging in other activities that require flexed postures for extended periods of time. When using a laptop computer, we can use a book to elevate the computer to keep the center line of the screen 
and the line of sight on a level surface, and we can lift the book or smart phone or other electronic products in front of us to reduce the possibility of flexed posture of cervical. We can also use an APP or a clock with timing and reminder functions, setting DCF to be less than 6 hours, or according to the actual situation of the individual, if using the smart phone for longer than the setting time, which can sound an alarm to remind you, or force the screen to turn off. Of course, these are all passive measures. The most important thing is that we need to develop good living and working habits. If not necessary, try not to lower our cervical to engage in activities, which may harmful for our cervical.

One limitation to our study was the lack of follow-up, therefore, the long-term results of our investigation were unknown. All recruitment filled out the questionnaire through self-reporting. Due to the use of online filling methods, and taking into account medical ethics rule, all participants were anonymous. We cannot ensure the validity of the survey content; this is a common limitation of survey design. And we cannot make sure the ratio of subjects suffering from cervical spondylosis before our survey, even though we adopted a random survey method, which may still affect the incidence of neck pain syndrome. We did not find suitable literature to extract the effect size for sample size estimation, so we cannot fully determine whether there are false positives and false negative situation. We did not analyze other risk factors associated with NDI scores such as mental stress, the time of day that people are working, working hours, work pressures, sleep quality, and financial situation. In the future, we hope to expand sample size to conduct further studies and evidence-based research on DCF and NDI scores.

\section{Abbreviations}

Cl: Confidence interval

CS: Cervical spondylosis

MRI: Magnetic resonance imaging

NDI: Neck Disability Index

NPDS: Neck Pain and Disability Scale

SD: Standard deviation

DCF: duration of cervical flexion per day

\section{Declarations}

Ethics approval and consent to participate: All participants filled out the questionnaire in anonymous way, the survey study need not approval by our Ethics Committee of hospital; informed consent was waived due to the nature of this survey study, which coincident with ethical requirements and waiver from the local research ethics committee. 
Consent for publication: Not applicable.

Availability of data and materials: The datasets analyzed during the current study are available from the corresponding author on reasonable request.

Funding: This research received no specific grant from any funding agency in the public, commercial, or not-for-profit sectors.

Competing interests: The authors declare that they have no conflict of interest.

Authors' contributions: JLZ: Conducted the study. Collected, analyzed and interpreted the data. Wrote the manuscript. YYF: Designed the study. Interpreted the data. Edited the manuscript.LJG: Statistical analyzed and interpreted the data. JC and CW: Created and statistical analyzed the data. $\mathrm{HCH}$ : Planned the project. Reviewed the manuscript. QLL: Planned the project. Reviewed the manuscript. Final approval of the version to be submitted: JLZ, YYF, LJG,JC, CW, HCH,QLL. The author(s) read and approved the final manuscript

Acknowledgements: We sincerely thank Dechao Xu for collecting and sorting participants' information. We also thank Zeling Tao and Wenxiang Fan for devising the questionnaire using Questionnaire Star. We thank the participants in the study for their time and effort. We thank Edanz Group China (www.liwenbianji.cn/ac), and Editage (www.editage.com) for English language editing for editing the English text of a draft of this manuscript.

\section{References}

1. Malige A, Morton PN, Carolan GF, Sokunbi G: The operative treatment of shoulder pain in patients with a concurrent diagnosis of cervical spondylosis and shoulder dysfunction. Journal of spine surgery 2019, 5(2):207-214.

2. Zheng C, Nie C, Zhu Y, Yu Q, Zhu D, Lu F, Weber R, Jiang J: Changes in Central Motor Conduction Time and Its Implication on Dysfunction of Distal Upper Limb in Distal-Type Cervical Spondylotic Amyotrophy. Journal of clinical neurophysiology : official publication of the American Electroencephalographic Society 2019, 36(1):52-59.

3. D MK, B EA: Diagnostic criteria for distinguishing cervical disk herniation from spondylosis in the neural compression syndrome. Radiology 1964, 83:67-73.

4. Woodworth DC, Holly LT, Mayer EA, Salamon N, Ellingson BM: Alterations in Cortical Thickness and Subcortical Volume are Associated With Neurological Symptoms and Neck Pain in Patients With Cervical Spondylosis. Neurosurgery 2019, 84(3):588-598.

5. Venosa M, Romanini E, Padua R, Cerciello S: Comparison of high-intensity laser therapy and combination of ultrasound treatment and transcutaneous nerve stimulation in patients with cervical spondylosis: a randomized controlled trial. Lasers in medical science 2019, 34(5):947-953. 
6. Stocks SJ, McNamee R, van der Molen HF, Paris C, Urban P, Campo G, Sauni R, Martinez Jarreta B, Valenty $\mathrm{M}$, Godderis $\mathrm{L}$ et al: Trends in incidence of occupational asthma, contact dermatitis, noiseinduced hearing loss, carpal tunnel syndrome and upper limb musculoskeletal disorders in European countries from 2000 to 2012. Occupational and environmental medicine 2015, 72(4):294-303.

7. Cook C, Richardson JK, Braga L, Menezes A, Soler X, Kume P, Zaninelli M, Socolows F, Pietrobon R: Cross-Cultural Adaptation and Validation of the Brazilian Portuguese Version of the Neck Disability Index and Neck Pain and Disability Scale. Spine, 31(14):1621-1627.

8. Ackelman $\mathrm{BH}$, Lindgren $\mathrm{U}$ : Validity and reliability of a modified version of the neck disability index. Journal of Rehabilitation Medicine 2002, 34(6):284-287.

9. Pietrobon R, Coeytaux RR, Carey TS, Richardson WJ, Devellis RF: Standard Scales for Measurement of Functional Outcome for Cervical Pain or Dysfunction. Spine, 27(5):515-522.

10. Jovicic MD, Konstantinovic LM, Grgurevic AD, Milovanovic ND, Trajkovic G, Jovicic VZ, Kostic Dedic SI, Hrkovic MK, Draganac SM: Validation of the Neck Disability Index in Serbian Patients With Cervical Radiculopathy. Journal of manipulative and physiological therapeutics 2018, 41(6):496-502.

11. Wheeler AH, Goolkasian P, Baird AC, Darden BV: Development of the Neck Pain and Disability Scale. Spine, 24(13):1290.

12. Stefanovitch-Lawbuary N, Amirfeyz R, Lovell R, Bannister G: Reliability and Responsiveness of Patient-Reported Outcome Measures of Neck Disability to Physical Therapy: Comparison of the Copenhagen, Northwick Park, and Neck Bournemouth Questionnaires and the Neck Disability Index. Journal of manipulative and physiological therapeutics 2019, 42(2):104-107.

13. Wolowich W, Casavant M, Ekins B: Phenylpropanolamine and hemorrhagic stroke. The New England journal of medicine 2001, 344(14):1094-1095.

14. Jaddoe V, de Jonge L, Hofman A, Franco O, Steegers E, Gaillard R: First trimester fetal growth restriction and cardiovascular risk factors in school age children: population based cohort study. BMJ (Clinical research ed) 2014, 348:g14.

15. Kolenkiewicz M, Wlodarczyk A, Wojtkiewicz J: Diagnosis and Incidence of Spondylosis and Cervical Disc Disorders in the University Clinical Hospital in Olsztyn, in Years 2011-2015. BioMed research international 2018, 2018:5643839.

16. Lv Y, Tian W, Chen D, Liu Y, Wang L, Duan F: The prevalence and associated factors of symptomatic cervical Spondylosis in Chinese adults: a community-based cross-sectional study. BMC Musculoskelet Disord 2018, 19(1):325.

17. Deng YZ, Xu LG, Chen L, Zhou D, Liu Y: Effectiveness of acupuncture in the management of cervical spondylosis: a meta-analysis. Journal of biological regulators and homeostatic agents 2017, 31(4):1017-1022.

18. Badhiwala JH, Witiw CD, Nassiri F, Jaja BNR, Akbar MA, Mansouri A, Merali Z, Ibrahim GM, Wilson JR, Fehlings MG: Patient phenotypes associated with outcome following surgery for mild degenerative cervical myelopathy: a principal component regression analysis. Spine J 2018, 18(12):2220-2231. 
19. Vernon H, Mior S: The Neck Disability Index: A Study of Reliability and Validity. Journal of manipulative and physiological therapeutics 1991, 14(7):409-415.

20. Breivik H: The Norwegian version of the Neck Disability Index (NDI) is reliable and sensitive to changes in pain-intensity and consequences of pain-in-the-neck. Scandinavian journal of pain 2014, 5(1):26-27.

21. Ko S, Choi W, Chae S, Kwon J, Lee Y: Correlation between Short-Form 36 Scores and Neck Disability Index in Patients Undergoing Anterior Cervical Discectomy and Fusion. Asian spine journal 2018, 12(4):691-696.

22. Feng Q, Liu S, Yang L, Xie M, Zhang QA-OhooX: The Prevalence of and Risk Factors Associated with Musculoskeletal Disorders among Sonographers in Central China: A Cross-Sectional Study. PloS one 2016, 11(10):1932-6203 (Electronic).

23. Gremark Simonsen J, Axmon A, Nordander C, Arvidsson I: Neck and upper extremity pain in sonographers - Associations with occupational factors. Applied Ergonomics 2017, 58:245-253.

24. Ando T: [Management of Cervical Spondylosis by Neurologists]. Brain and nerve = Shinkei kenkyu no shinpo 2019, 71(3):239-248.

25. Usmani S, Rasheed R, Al Kandari F: Textitis as Seen on F-NaF Imaging Using an Ultra-HighResolution Positron Emission Mammography Scanner. Journal of nuclear medicine technology 2020, 48(2):181-183.

26. Park J, Kang S, Lee S, Jeon $\mathrm{H}$ : The effects of smart phone gaming duration on muscle activation and spinal posture: Pilot study. Physiotherapy theory and practice 2017, 33(8):661-669.

27. Kim S, Koo S: Effect of duration of smartphone use on muscle fatigue and pain caused by forward head posture in adults. Journal of physical therapy science 2016, 28(6):1669-1672.

\section{Tables}

Table 1. Sociodemographic and clinical characteristics of the sample 


\begin{tabular}{llll}
\hline Sexes & \multicolumn{1}{c}{$\begin{array}{c}\text { Male } \\
\text { Mean+SD/N\% }\end{array}$} & $\begin{array}{c}\text { Female } \\
\text { Mean+SD/N\% }\end{array}$ & P-value \\
\hline N & 124 & 374 & \\
Age, years & $32.20 \pm 9.30$ & $31.44 \pm 7.17$ & 0.699 \\
Working age, years & $8.70 \pm 7.66$ & $9.10 \pm 6.45$ & 0.068 \\
DCF, hours & $5.85 \pm 3.13$ & $4.74 \pm 2.45$ & $<0.001^{*}$ \\
NDI & $6.17 \pm 4.48$ & $5.60 \pm 4.49$ & 0.139 \\
Educational degree & & & $<0.001^{*}$ \\
$\quad$ Junior school level or below & $4(3.23 \%)$ & $5(1.34 \%)$ & \\
$\quad$ High school level or below & $3(2.42 \%)$ & $2(0.53 \%)$ & \\
$\quad$ Bachelor's or below & $92(74.19 \%)$ & $348(93.05 \%)$ & \\
$\quad$ Master's or above & $25(20.16 \%)$ & $19(5.08 \%)$ & \\
Activities & & & \\
$\quad$ Smart phone use & $79(63.71 \%)$ & $292(78.07 \%)$ & \\
$\quad$ Using computers & $38(30.65 \%)$ & $70(18.72 \%)$ & \\
$\quad$ Reading books & $2(1.61 \%)$ & $8(2.14 \%)$ & \\
$\quad$ Using other electronic devices & $5(4.03 \%)$ & $4(1.07 \%)$ & \\
Low back pain & & & \\
$\quad$ No & $33(26.61 \%)$ & $82(21.93 \%)$ & \\
$\quad$ Yes & $91(73.39 \%)$ & $292(78.07 \%)$ & \\
Neck pain & & $126(33.69 \%)$ & \\
$\quad$ No & $32(25.81 \%)$ & $248(66.31 \%)$ & \\
$\quad$ Yes & $92(74.19 \%)$ & & \\
\hline
\end{tabular}

Note: DCF, duration of cervical flexion per day; NDI, Neck Disability Index; SD, standard deviation; ${ }^{*} P<0.05$.

Table 2. Crude correlation associations of DCF, covariates, and NDI scores of the sample

\begin{tabular}{llll}
\hline & Statistics & \multicolumn{1}{c}{ NDI scores } & P-value \\
\cline { 3 - 3 } & & \multicolumn{1}{c}{$\beta(95 \% \mathrm{CI})$} & \\
\hline Age, years & $31.63 \pm 7.76$ & $0.02(-0.03,0.07)$ & 0.4169 \\
Working age, 5 years & $9.01 \pm 6.76$ & $-0.01(-0.30,0.28)$ & 0.9451 \\
DCF, hours & $5.02 \pm 2.68$ & $0.28(0.13,0.42)$ & $0.0002^{*}$ \\
Educational degree & & & \\
$\quad$ Junior school level or below & $9(1.81 \%)$ & $0.25(-2.71,3.21)$ & 0.8683 \\
High school level or below & $5(1.00 \%)$ & $3.96(0.01,7.91)$ & $0.0499^{*}$ \\
Bachelor's or below & $440(88.35 \%)$ & 0 & \\
$\quad$ Master's or above & $44(8.84 \%)$ & $0.68(-0.71,2.07)$ & 0.3379 \\
Activities & & & \\
$\quad$ Others & $371(74.50 \%)$ & 0 & \\
$\quad$ Smart phone use & $127(25.50 \%)$ & $0.83(-0.07,1.73)$ & 0.0716 \\
Low back pain & & & \\
$\quad$ No & $115(23.09 \%)$ & 0 & \\
$\quad$ Yes & $383(76.91 \%)$ & $2.40(1.49,3.31)$ & $<0.0001^{*}$ \\
\hline
\end{tabular}

Note: DCF, duration of cervical flexion per day; NDI, Neck Disability Index; CI, confidence interval; $*$ P $<0.05$.

Table 3. Linear regression model for DCF and NDI scores

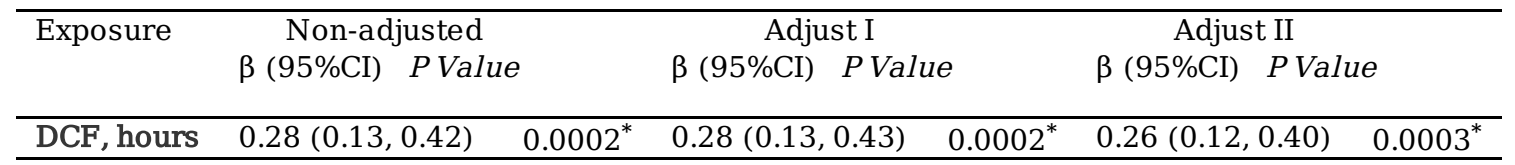

Note: Non-adjusted model adjusted for: None; Adjust I model adjust for: age, sex, and working age; Adjust II model adjusted for low back pain; ${ }^{*} P<0.05$.

Table 4. Analysis of threshold saturation effect between DCF and NDI scores 


\begin{tabular}{|c|c|c|}
\hline Outcome & $\begin{array}{r}\text { NDI } \\
\beta 95 \% \mathrm{CI}\end{array}$ & $P$ Value \\
\hline \multicolumn{3}{|l|}{ Model $\square$} \\
\hline $\begin{array}{l}\text { A linear regression coefficient } \\
\text { Model } \square\end{array}$ & $0.26(0.12,0.41)$ & $0.0005^{*}$ \\
\hline Fold point $(\mathrm{K})$ & 6 & \\
\hline$<\mathrm{K}$ regression coefficient 1 & $0.53(0.26,0.81)$ & $0.0002^{*}$ \\
\hline$>\mathrm{K}$ regression coefficient 2 & $-0.03(-0.33,0.26)$ & 0.8225 \\
\hline Logarithmic likelihood ratio test & & $0.023^{*}$ \\
\hline
\end{tabular}

Note: ${ }^{*} P<0.05$.

Figures

1791 participants came from nationwide Age from 15-75 and filled the questionnaire online between May 29, 2019 to April 10, 2020

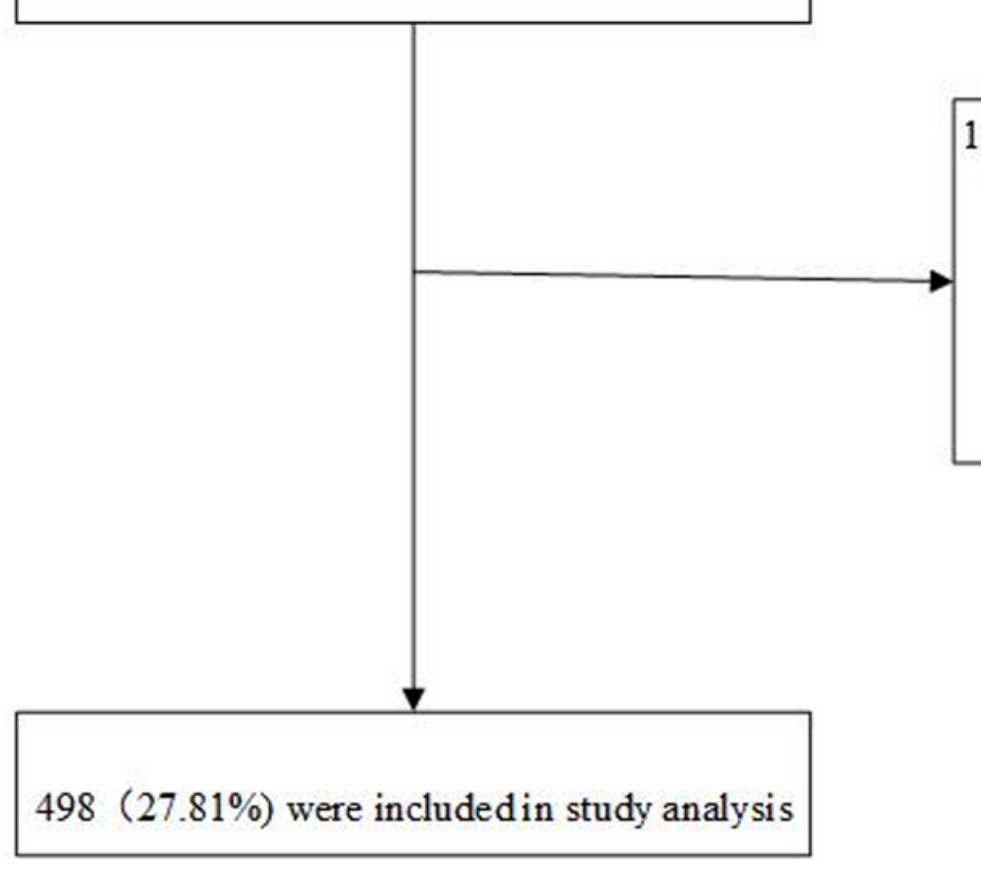

1293 participants excluded( 5 were under 18 years) $563(31.34 \%)$ individuals did not complete the questionnaire $730(40.76 \%)$ individuals were no office workers, including $218(12.17 \%)$ students, $78(4.36 \%)$ building workers, $434(24.23 \%)$ other nonoffice workers

\section{Figure 1}

Flow chart of this study 


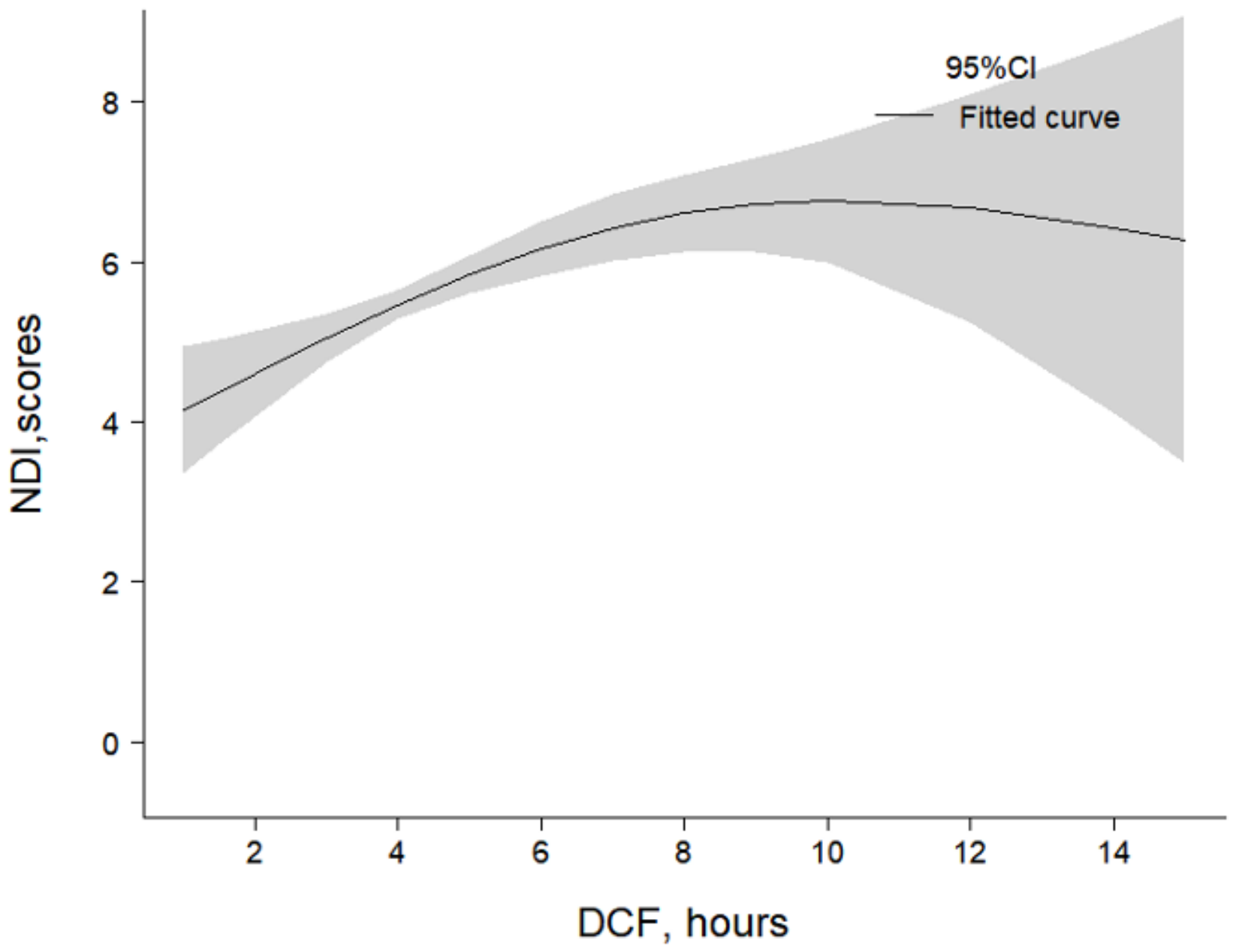

Figure 2

General additive models demonstrate the relationship between DCF and the NDI scores. The resulting figures showed the predicted NDI scores in the y-axis and the DCF in the x-axis.

\section{Supplementary Files}

This is a list of supplementary files associated with this preprint. Click to download.

- SupplementalMaterial.doc 